(6) OPEN ACCESS

\title{
The safety of triple antiplatelet therapy under thromboelastography guidance in patients undergoing stenting for ischemic cerebrovascular disease
}

\author{
Zhonghua Wu, ${ }^{1,2,3}$ Ao-Fei Liu, ${ }^{2}{ }^{2}$ Z Zhou, ${ }^{2}$ Yiqun Zhang, ${ }^{2}$ Kai Wang, ${ }^{2}$ Chen Li, ${ }^{2}$ \\ Hancheng Qiu, ${ }^{2}$ Wei-Jian Jiang ${ }^{1,2}$
}

${ }^{1}$ General Hospital of the PLA Rocket Force, The Teaching Hospital of Soochow University, Suzhou, China

${ }^{2}$ Department of Vascular Neurosurgery, New Era Stroke Care and Research Institute, General Hospital of the PLA Rocket Force, Beijing, China ${ }^{3}$ Department of Neurosurgery, The Sixth People's Hospital of Nantong, Nantong, China

\section{Correspondence to} Dr. Wei-Jian Jiang, General Hospital of the PLA Rocket Force, The Teaching Hospital of Soochow University, Suzhou, China; Department of Vascular Neurosurgery, New Era Stroke Care and Research Institute, General Hospital of the PLA Rocket Force, Beijing 100088, China.; jiangweijian2018@ 163.com

ZW and A-FL contributed equally.

Received 4 April 2018 Revised 24 July 2018 Accepted 26 July 2018 Published Online First 12 August 2018

\section{ABSTRACT \\ Objective To investigate the safety of triple antiplatelet therapy (TAT) with cilostazol in patients undergoing stenting for extracranial and/or intracranial artery stenosis.}

Methods A prospectively collected database was reviewed to identify patients who underwent stenting for extracranial and/or intracranial artery stenosis and showed resistance to aspirin and/or clopidogrel as assessed by pre-stenting thromboelastography (TEG) testing. Patients were assigned to a TAT group and a dual antiplatelet therapy (DAT) group. Major complications were defined as thromboembolic events (transient ischemic attack (TIA), ischemic stroke, and stent thrombosis) or major bleeding events within 30 days, and minor complications were defined as extracranial bleeding that did not require vascular surgery or transfusion within 30 days.

Results A total of 183 patients were identified. The incidence of major complications was significantly lower in the TAT group than in the DAT group (TAT group vs. DAT group, $1 / 110$ vs. $6 / 73 ; P=0.017$ ). TIAs occurred in four patients, with one in the TAT group and three in the DAT group (1/110 vs. 3/73; $P=0.303$ ). Ischemic strokes occurred in three patients in the DAT group (TAT group vs. DAT group, $\mathrm{P}=0.062$ ). No major bleeding events or stent thrombosis was recorded in either group. Two patients (one in each group) experienced minor complications that resolved without additional treatment (1/110 vs. $1 / 73 ; P>0.999)$.

Conclusions TAT under TEG guidance appears to be a safe antiplatelet strategy in patients undergoing stenting for extracranial and/or intracranial artery stenosis. By employing TAT under TEG guidance, favorable outcomes can be achieved in these patients.

\section{INTRODUCTION}

Stent implantation for extracranial and/or intracranial artery stenosis is technically feasible but associated with high rates of periprocedural ischemic complications. ${ }^{12}$ Dual antiplatelet therapy (DAT; aspirin plus clopidogrel) is a standard perioperative preparation for neurovascular stent placement to prevent thromboembolic events. ${ }^{3} 4$ However, the incidence of thromboembolic events remains unacceptable. ${ }^{5}$ In multiple studies on percutaneous coronary intervention, the occurrence of thromboembolic events is associated with resistance to antiplatelet drugs, ${ }^{67}$ and triple antiplatelet therapy (TAT) with cilostazol is a safe and effective alternative. ${ }^{8} 9$ However, thus far, little information has been available regarding TAT in patients undergoing stenting for ischemic cerebrovascular disease, largely due to the perceived risk of increased bleeding complications, especially intracranial hemorrhage, when antithrombotic effects are intensified.

Some studies have reported the reliability and convenience of thromboelastography (TEG) in assessing platelet function. ${ }^{10}{ }^{11}$ However, neurovascular stenting data are limited. Therefore, the present study sought to evaluate the safety of TAT under TEG guidance in patients undergoing extracranial and/or intracranial artery stenting.

\section{METHODS}

\section{Study population}

Patients were selected from a prospectively maintained database at our center between January 2013 and June 2017. Patients who had undergone stenting for extracranial and/or intracranial atherosclerotic stenosis and showed resistance to aspirin and/or clopidogrel (reported by pre-stenting TEG) were eligible for inclusion in this study, whereas those who did not comply with our standard dual antiplatelet preparation were excluded. Figure 1 is a flowchart showing the process of patient selection. Informed consent was obtained from all patients, and the protocol was approved by the Institutional Review Board of the General Hospital of the PLA Rocket Force.

All procedures were performed by an experienced interventional neurointerventionist (WJJ), and all patients received strict perioperative management. Patients' demographic and clinical characteristics, site and number of stents, antiplatelet regimen, and thromboembolic and hemorrhagic complications within 30 days after stenting were identified and recorded.

\section{Medications and platelet function testing}

All patients received the standard dual antiplatelet preparation $(300 \mathrm{mg}$ aspirin and $75 \mathrm{mg}$ clopidogrel daily) for at least 5 days before the stenting procedure. Platelet function was tested by using a TEG 
437 patients with pre-stenting TEG test and received stenting for extracranial and/or intracranial atherosclerotic stenosis

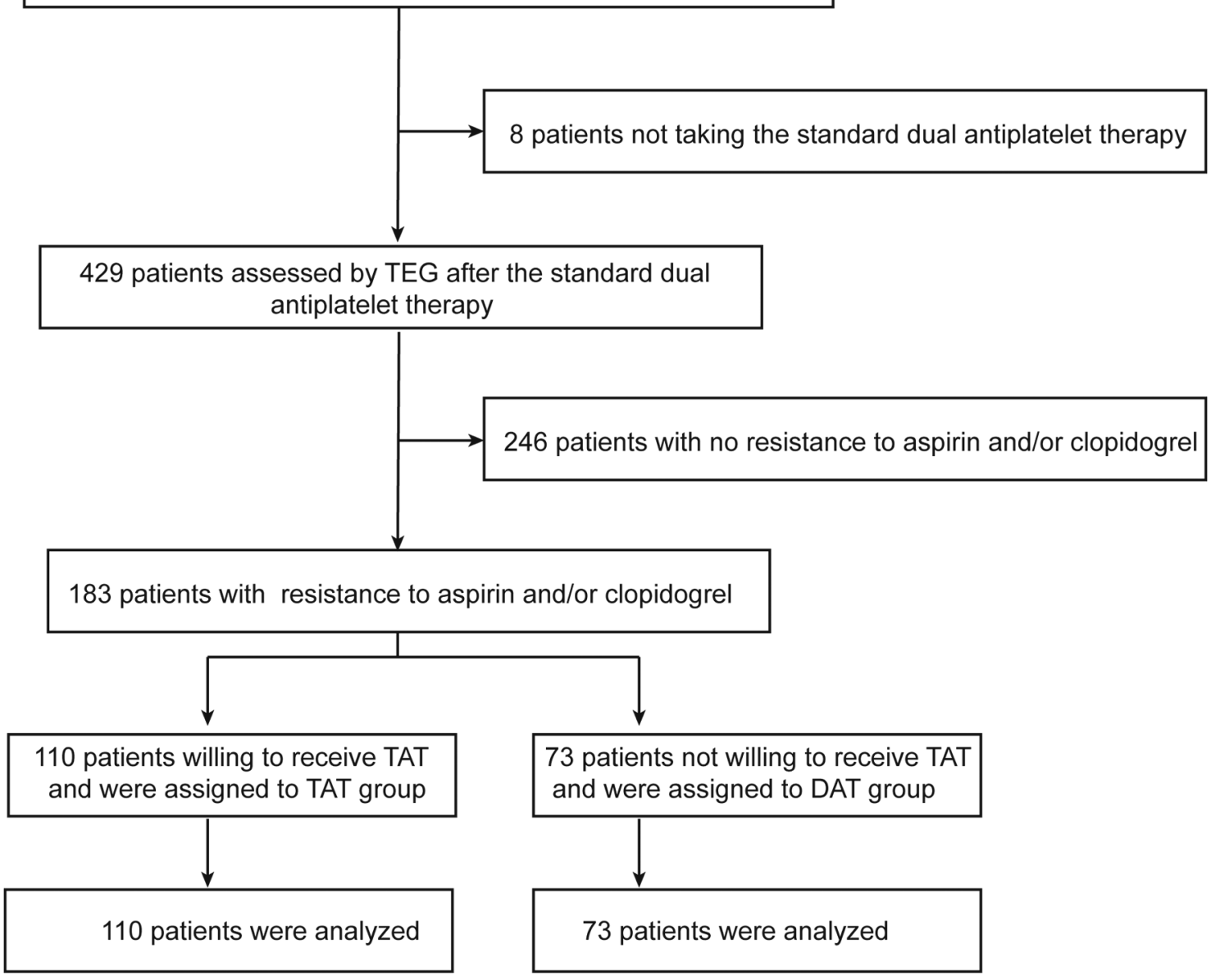

Figure 1 The flowchart summarizing the process of patient selection.

Haemostasis System (Model YZ5000; Shanxi Yu Ze Yi Medical Co., Ltd., Shanxi, China) the day before the procedure. Two TEG parameters, arachidonic acid inhibition rate (AA\%) and ADP inhibition rate (ADP\%), were collected. An AA\% less than $50 \%$ and an ADP $\%$ less than $50 \%$ were defined as resistance to aspirin and clopidogrel, respectively. Patients with aspirin and/or clopidogrel resistance were placed into the TAT or DAT groups, respectively, based on informed patient choice. Patients in the DAT group received $300 \mathrm{mg}$ aspirin and $75 \mathrm{mg}$ clopidogrel daily. Patients in the TAT group received $300 \mathrm{mg}$ aspirin and $75 \mathrm{mg}$ clopidogrel daily and $100 \mathrm{mg}$ cilostazol twice daily: a dose of $200 \mathrm{mg}$ cilostazol was administered at least 12 hours before stenting.

After discharge, the aspirin dose for all patients was changed to $100 \mathrm{mg}$ daily. For patients in the TAT group, cilostazol was withdrawn 4 weeks after stenting. Three months after the procedure, a single antiplatelet agent $(100 \mathrm{mg}$ aspirin or $75 \mathrm{mg}$ clopidogrel daily) was prescribed for each patient as a life-long maintenance therapy.

\section{Intraoperative antithrombotic process}

After placement of the guiding catheter, a bolus of heparin (3000 U) followed by an infusion of heparin at $800 \mathrm{U} /$ hour was administered intravenously according to our previous protocol. ${ }^{12}$ After ruling out intracranial hemorrhage by a post-stenting CT scan of the brain, an intravenous infusion of heparin (500 U/hour) was continued, and low-molecular-weight heparin (5000 IU;
Fragmin P forte 5000 IE; Pharmacia, Peapack, NJ) was administered subcutaneously every 12 hours for 3 days starting at $8 \mathrm{pm}$ on the day of surgery. If acute stent thrombosis was confirmed during surgery or if subacute thrombosis was confirmed, tirofiban (Grand Pharmaceutical Co., Ltd., Wuhan, China) was administered intravenously or intraarterially.

\section{Major and minor complications}

Major complications included thromboembolic or major bleeding events within 30 days. A thromboembolic event was defined as a transient ischemic attack (TIA), ischemic stroke, or stent thrombosis occurring in the region of the stented artery. A major bleeding event was defined as intracranial hemorrhage or extracranial bleeding requiring surgery or transfusion. Minor complications were defined as extracranial bleeding that did not require vascular surgery or transfusion. An emergent brain MRI scan was routinely performed if a new neurologic symptom developed after stenting. Two stroke neurologists blinded to the study evaluated and recorded the complications. Outpatient or telephone follow-up of all patients was performed at 30 days after hospital discharge, and the occurrence of adverse events was identified.

\section{Statistical analysis}

Continuous variables were compared using Student's $t$-test or the Wilcoxon rank-sum test. Categorical variables were compared using Pearson's chi-square test or Fisher's exact test. Continuous 
variables were expressed as mean \pm SD deviation (SD) or median and IQR according to the sample distribution. Categorical variables were expressed as absolute numbers (n) and percentage $(\%)$. Differences were considered statistically significant at Pvalues $<0.05$ (two-tailed). Statistical analysis was performed with SPSS 21.0 software (IBM SPSS, Armonk, NY).

\section{RESULTS}

Between January 2013 and June 2017, a total of 429 patients with extracranial and/or intracranial atherosclerotic stenosis were treated with a stent procedure: all complied with our standard dual antiplatelet preparation and completed TEG before stenting. As a result, 183 patients with resistance to aspirin and/or clopidogrel were enrolled in the study. The rates of resistance to aspirin, clopidogrel, or both as revealed by TEG were 5.1\% (22/429), $40.6 \%$ (174/429), and 3.0\% (13/429), respectively. Among the 183 patients who were ultimately enrolled, 110 (60.1\%) received
TAT (plus cilostazol $100 \mathrm{mg}$ twice daily), while the remaining 73 $(39.9 \%)$ received DAT. There were no significant differences in the baseline characteristics between the two groups (table 1).

In the present study, seven thromboembolic events (four TIAs and three ischemic strokes) were recorded, and all occurred within 3 days after stenting. The rate of major complications was $0.9 \%(1 / 110)$ in the TAT group and $8.2 \%(6 / 73)$ in the DAT group (OR, 0.102; 95\% CI, 0.012 to $0.870 ; \mathrm{P}=0.017$ ). One patient in the TAT group and three patients in the DAT group experienced TIAs $(\mathrm{P}=0.303)$. ischemic stroke, as demonstrated by MRI, occurred in three patients in the DAT group vs. none in the TAT group (TAT group vs. DAT group, $\mathrm{P}=0.062$ ). No major bleeding events or stent thrombosis were recorded. Two patients (one in each group) experienced minor complications (gastrointestinal bleeding) that resolved without additional treatment ( $1 / 110$ vs. $1 / 73 ; P>0.999)$. Table 2 shows the major and minor complications observed in the study.

Table 1 Baseline characteristics of the two study groups (TAT group and DAT group)

\begin{tabular}{|c|c|c|c|}
\hline & TAT group $(n=110)$ & DAT group $(n=73)$ & $\mathbf{P}$-values \\
\hline Age, mean $\pm S D$, years & $61.41 \pm 12.06$ & $60.27 \pm 9.88$ & 0.505 \\
\hline Male, n (\%) & $90(81.8 \%)$ & $53(72.6 \%)$ & 0.140 \\
\hline $\mathrm{BMI}$, mean $\pm \mathrm{SD}, \mathrm{kg} / \mathrm{m}^{2}$ & $25.16 \pm 2.80$ & $26.03 \pm 3.40$ & 0.060 \\
\hline Smoking, n (\%) & $52(47.3 \%)$ & $39(53.4 \%)$ & 0.415 \\
\hline CHD, n (\%) & $16(14.5 \%)$ & $16(21.9 \%)$ & 0.199 \\
\hline Diabetes, n (\%) & $38(34.5 \%)$ & $30(41.1 \%)$ & 0.369 \\
\hline Hypertension, n (\%) & $76(69.1 \%)$ & $54(74.0 \%)$ & 0.476 \\
\hline Prior cerebral infarction, n (\%) & $82(74.5 \%)$ & $49(67.1 \%)$ & 0.276 \\
\hline Hyperlipidemia, n (\%) & $43(39.1 \%)$ & $28(38.4 \%)$ & 0.920 \\
\hline Alcohol consumption, n (\%) & $32(29.1 \%)$ & $23(31.5 \%)$ & 0.727 \\
\hline \multicolumn{4}{|l|}{ Laboratory data } \\
\hline $\mathrm{TC}$, mean $\pm \mathrm{SD}, \mathrm{mmol} / \mathrm{L}$ & $3.40 \pm 0.88$ & $3.64 \pm 1.04$ & 0.095 \\
\hline $\mathrm{PLT}$, mean $\pm \mathrm{SD}, \times 10^{12} / \mathrm{L}$ & $211.46 \pm 66.68$ & $206.82 \pm 50.65$ & 0.614 \\
\hline ESR, median (IQR), mm/h & $6.00(4.00-10.00)$ & $7.00(3.75-14.00)$ & 0.406 \\
\hline CRP, median (IQR), mg/L & $1.57(0.68-3.35)$ & $1.90(1.01-4.27)$ & 0.093 \\
\hline $\mathrm{HCY}$, median (IQR), mmol/L & 12.45 (10.67-18.95) & $11.70(8.55-17.20)$ & 0.072 \\
\hline $\mathrm{LDL}$, mean $\pm \mathrm{SD}, \mathrm{mmol} / \mathrm{L}$ & $2.10 \pm 0.72$ & $2.13 \pm 0.95$ & 0.831 \\
\hline $\mathrm{PT}$, mean $\pm \mathrm{SD}, \mathrm{s}$ & $11.22 \pm 2.30$ & $10.74 \pm 0.73$ & 0.088 \\
\hline INR, mean \pm SD & $0.99 \pm 0.20$ & $0.95 \pm 0.07$ & 0.089 \\
\hline APTT, mean $\pm S D, s$ & $32.29 \pm 3.33$ & $32.38 \pm 3.20$ & 0.861 \\
\hline $\mathrm{GLU}$, mean $\pm \mathrm{SD}, \mathrm{mmol} / \mathrm{L}$ & $6.10 \pm 1.86$ & $6.02 \pm 1.46$ & 0.747 \\
\hline $\mathrm{HBA} 1 \mathrm{C}$, mean $\pm \mathrm{SD}, \%$ & $6.36 \pm 1.33$ & $6.24 \pm 0.84$ & 0.508 \\
\hline $\mathrm{RBC}$, mean $\pm \mathrm{SD}, \times 10^{12} / \mathrm{L}$ & $4.51 \pm 0.47$ & $4.49 \pm 0.50$ & 0.842 \\
\hline \multicolumn{4}{|l|}{ Antiplatelet resistance } \\
\hline Aspirin, n (\%) & $10(9.1 \%)$ & $12(16.4 \%)$ & 0.135 \\
\hline Clopidogrel, n (\%) & $106(96.4 \%)$ & $68(93.2 \%)$ & 0.487 \\
\hline Both, n (\%) & $6(5.5 \%)$ & $7(9.6 \%)$ & 0.320 \\
\hline \multicolumn{4}{|l|}{ Stent features } \\
\hline Intracranial stent, n (\%) & $62(56.4 \%)$ & $42(57.5 \%)$ & 0.876 \\
\hline Extracranial stent, n (\%) & $52(47.3 \%)$ & $37(50.7 \%)$ & 0.651 \\
\hline Both, n (\%) & $4(3.6 \%)$ & $6(8.2 \%)$ & 0.316 \\
\hline Number of stents $\geq 2, \mathrm{n}(\%)$ & $15(13.6 \%)$ & $14(19.2 \%)$ & 0.315 \\
\hline
\end{tabular}

APTT, activated partial thromboplastin time; BMI, body mass index; CHD, coronary heart disease; CRP, C-reactive protein; DAT, dual antiplatelet therapy; ESR, erythrocyte sedimentation rate; GLU, glucose; HbA1c, glycosylated hemoglobin; HCY, homocysteine; INR, International normalized ratio; LDL-C, low-density lipoprotein cholesterol; PLT, platelet; PT, prothrombin time; RBC, red blood cells; TAT, triple antiplatelet therapy; TC, total cholesterol. 
Table 2 Major and minor complications within 30 days (TAT vs DAT group)

\begin{tabular}{|c|c|c|c|c|c|}
\hline & TAT group $(n=110)$ & DAT group $(n=73)$ & P-values & OR & $95 \% \mathrm{Cl}$ \\
\hline Major complications, n (\%) & $1(0.9 \%)$ & $6(8.2 \%)$ & 0.017 & 0.102 & 0.012 to 0.870 \\
\hline TIA & $1(0.9 \%)$ & $3(4.1 \%)$ & 0.303 & 0.214 & 0.022 to 2.099 \\
\hline ischemic stroke & $0(0 \%)$ & $3(4.1 \%)$ & 0.062 & 1.043 & 0.994 to 1.094 \\
\hline Stent thrombosis & $0(0 \%)$ & $0(0 \%)$ & - & - & - \\
\hline Intracranial bleeding & $0(0 \%)$ & $0(0 \%)$ & - & - & - \\
\hline Extracranial bleeding & $0(0 \%)$ & $0(0 \%)$ & - & - & - \\
\hline Minor complications, n (\%) & $1(0.9 \%)$ & $1(1.4 \%)$ & $>0.999$ & 0.661 & 0.041 to 10.73 \\
\hline
\end{tabular}

DAT, dual antiplatelet therapy; TAT, triple antiplatelet therapy; TIA, transient ischemic attack.

\section{DISCUSSION}

The results of this study show that TAT under TEG guidance is a safe antiplatelet strategy that can reduce periprocedural thromboembolic events without increasing bleeding events in patients undergoing extracranial and/or intracranial artery stenting. Our results provide preliminary evidence for the safety and efficacy of the periprocedural use of TAT in neurointervention. Furthermore, to our best knowledge, this study represents the first and largest case series regarding these types of patients and procedures.

There is evidence in the cardiovascular and neurovascular literature that antiplatelet resistance is closely associated with the occurrence of periprocedural thrombotic events. ${ }^{13-17}$ Some recent studies, especially on cardiovascular interventions, have reported modified antiplatelet strategies for addressing antiplatelet resistance. However, to date, there has been no consensus regarding the optimal treatment strategy. Increasing the dose of antiplatelet drugs may decrease antiplatelet resistance to some extent, however high doses are insufficient to prevent thromboembolic events ${ }^{18}{ }^{19}$ and may lead to an increased risk of bleeding. ${ }^{19}{ }^{20}$ Novel potent P2Y12 inhibitors, such as prasugrel and ticagrelor, may represent promising choices for overcoming clopidogrel resistance, however whether the benefits of decreasing thromboembolic events can offset the risk of bleeding complications remains unclear. ${ }^{2122}$ Other antiplatelet strategies, such as adding cilostazol to DAT (as in TAT), may provide an additional benefit: this strategy is a reportedly safe and effective antiplatelet regimen for cardiovascular interventions. ${ }^{172324}$ However, because TAT might increase the risk for a major bleeding event (especially intracranial bleeding) while intensifying the antithrombotic effect, interventional neuroradiologists are hesitant to adopt TAT to manage antiplatelet resistance. Therefore, evaluating the safety of TAT in neurointervention is significant and useful.

The present study focused on the safety of TAT during the perioperative management of patients undergoing extracranial and/or intracranial artery stent implantation. Our results show that compared with the DAT group, the TAT group had a reduction in the rate of thromboembolic events without an increased risk of bleeding. This finding is consistent with large-scale clinical research on cardiovascular interventions. ${ }^{8} 2526$ However, to our knowledge, only two studies have evaluated the clinical performance of TAT with the monitoring of platelet function testing (PFT) in neurointervention. One prospective randomized single-center study of stent-assisted coil embolization for unruptured intracranial aneurysms ${ }^{3}$ demonstrated that the group of patients who received a modified antiplatelet preparation (mainly with the addition of cilostazol) had a lower rate of thromboembolic events than did the group of patients who received the standard preparation $(1.6 \%$ vs. $11.1 \% ; \mathrm{P}=0.02)$ without an increased risk of bleeding events. In another study involving patients with resistance to clopidogrel undergoing carotid artery stenting, TAT decreased the number of ischemic lesions detected by diffusion-weighted imaging (TAT group vs. DAT group; $9.1 \%$ vs $25 \%$ ) without any increase in bleeding events. $^{27}$

In our opinion, the following possible explanations may account for the absence of an overall increased risk of bleeding in the TAT group: the use of TEG guidance; the low bleeding risk associated with cilostazol as demonstrated by the Cilostazol Stroke Prevention Study 2 (CSPS 2);28 the potential protective effects of cilostazol on endothelial cells and the blood-brain barrier $^{; 2}$ and the short-term duration of TAT. In the Stenting versus Aggressive Medical Management for Preventing Recurrent Stroke in Intracranial Stenosis (SAMMPRIS) trial, the rate of intracranial hemorrhage was $5.8 \%$ in the intervention cohort (a total of 224 patients) within 30 days, including seven parenchymal hemorrhages and six subarachnoid hemorrhages. In contrast, no intracranial hemorrhages were observed in the present study. Detailed analysis ${ }^{30}$ of the SAMMPRIS trial indicated that hemorrhagic stroke was associated with clopidogrel load, hyperperfusion injury, and operator experience, which is consistent with our efforts regarding stenting of cerebral artery stenosis. In the present study, we attribute our favorable results to the adoption of an appropriate antithrombotic therapy, strict perioperative management (eg, stroke risk factors and postprocedural blood pressure), a customized stenting procedure based on the characteristics of the target lesion (eg, plaque distribution and angulation), and the participation of an experienced neuroradiologist who had completed at least 600 intracranial and 2000 extracranial stent placement procedures between 2001 and 2013.

In most cardiovascular trials, an ADP\% less than 30\% as assessed by TEG is defined as clopidogrel resistance, nevertheless this issue remains somewhat controversial. ${ }^{17}{ }^{31}$ Considering the potentially high rate of periprocedural thromboembolic events in the setting of extracranial and/or intracranial artery stent implantation and the low bleeding risk associated with cilostazol, ${ }^{28}$ a higher cut-off value of $50 \%$ was used to define clopidogrel resistance in this study. The higher cut-off value for ADP\% did not lead to a high bleeding risk, which was similar to the results of a previous study. ${ }^{3}$ Hence, our data suggest that adopting this higher threshold for clopidogrel resistance in neurointervention is feasible and safe. Thus far, PFT has not been routinely used in neurointervention owing to the lack of a consensus on the optimal method and cut-off value. Nevertheless, if PFT is available, we recommend utilizing PFT before the procedure and then modifying the antiplatelet regimen based on its results. Furthermore, we advocate the collection and publication of data by all relevant centres to further our understanding 
of the role of PFT and PFT-guided antiplatelet regimens in neurointervention.

\section{LIMITATIONS}

This study has several limitations. First, this was a single-center, nonrandomized study, and the study population comprised Chinese patients only. Whether TAT under TEG guidance would have similar effects in other ethnic groups is uncertain. Second, a second TEG test was not performed after the addition of cilostazol. Third, silent infarction was not considered in this study because routine MRI evaluation was not performed after stent implantation. Fourth, not all possible factors associated with periprocedural complications could be considered. Finally, the sample size was not sufficiently large taking into account our relatively low rate of perioperative complications. A large multicenter, prospective, randomised controlled trial is necessary to further validate the present results.

\section{CONCLUSION}

TAT under TEG guidance is safe and can reduce the incidence thromboembolic events without a high risk of bleeding in patients undergoing stenting for extracranial and/or intracranial artery stenosis. Hence, we advocate the use of TAT under PFT guidance in these patients.

Acknowledgements We would like to thank Li Xiang, Department of Record Room, the General Hospital of the PLA Rocket Force, Beijing, 100088, China, for her help in providing data.

Contributors W-JJ contributed the conception, design, data analysis, and manuscript revision. ZW and A-FL collected the data and drafted the manuscript. JZ and $Y Z$ : analyzed data and revised the manuscript. $\mathrm{KW}, \mathrm{CL}$, and $\mathrm{HQ}$ : reviewed and edited the manuscript.

Funding The authors have not declared a specific grant for this research from any funding agency in the public, commercial, or not-for-profit sectors.

Competing interests None declared.

Patient consent Not required.

Ethics approval This study was approved by the institutional ethics committee at the General Hospital of the PLA Rocket Force.

Provenance and peer review Not commissioned; externally peer reviewed.

Open access This is an open access article distributed in accordance with the Creative Commons Attribution Non Commercial (CC BY-NC 4.0) license, which permits others to distribute, remix, adapt, build upon this work non-commercially, and license their derivative works on different terms, provided the original work is properly cited, appropriate credit is given, any changes made indicated, and the use is non-commercial. See: http://creativecommons.org/licenses/by-nc/4.0/.

\section{REFERENCES}

1 Roubin GS, New G, lyer SS, et al. Immediate and late clinical outcomes of carotid artery stenting in patients with symptomatic and asymptomatic carotid artery stenosis: a 5-year prospective analysis. Circulation 2001;103:532-7.

2 Jiang WJ, Du B, Leung TW, et al. Symptomatic intracranial stenosis: cerebrovascular complications from elective stent placement. Radiology 2007;243:188-97.

3 Hwang G, Huh W, Lee JS, et al. Standard vs modified antiplatelet preparation for preventing thromboembolic events in patients with high on-treatment platelet reactivity undergoing coil embolization for an unruptured intracranial aneurysm: a randomized clinical trial. JAMA Neurol 2015;72:764-72.

4 Gandhi CD, Bulsara KR, Fifi J, et al. Platelet function inhibitors and platelet function testing in neurointerventional procedures. J Neurointerv Surg 2014;6:567-77.

5 Fifi JT, Brockington C, Narang J, et al. Clopidogrel resistance is associated with thromboembolic complications in patients undergoing neurovascular stenting. AJNR Am J Neuroradiol 2013;34:716-20.

6 Chen WH, Lee PY, $\mathrm{Ng} \mathrm{W}$, et al. Aspirin resistance is associated with a high incidence of myonecrosis after non-urgent percutaneous coronary intervention despite clopidogrel pretreatment. J Am Coll Cardiol 2004:43:1122-6.
7 Mehta SR, Yusuf S, Peters RJ, et al. Effects of pretreatment with clopidogrel and aspirin followed by long-term therapy in patients undergoing percutaneous coronary intervention: the PCI-CURE study. Lancet 2001;358:527-33.

8 Lee SW, Park SW, Hong MK, et al. Triple versus dual antiplatelet therapy after coronary stenting: impact on stent thrombosis. J Am Coll Cardiol 2005;46:1833-7.

9 Chen KY, Rha SW, Li YJ, et al. Triple versus dual antiplatelet therapy in patients with acute ST-segment elevation myocardial infarction undergoing primary percutaneous coronary intervention. Circulation 2009;119:3207-14.

10 Gurbel PA, Bliden KP, Navickas IA, et al. Adenosine diphosphate-induced plateletfibrin clot strength: a new thrombelastographic indicator of long-term poststenting ischemic events. Am Heart J 2010;160:346-54.

11 Cattano D, Altamirano AV, Kaynak HE, et al. Perioperative assessment of platelet function by thromboelastograph platelet mapping in cardiovascular patients undergoing non-cardiac surgery. J Thromb Thrombolysis 2013;35:23-30.

12 Jiang WJ, Wang YJ, Du B, et al. Stenting of symptomatic M1 stenosis of middle cerebral artery: an initial experience of 40 patients. Stroke 2004;35:1375-80.

13 Wang B, Li XQ, Ma N, et al. Association of thrombelastographic parameters with poststenting ischemic events. J Neurointerv Surg 2017;9:192-5.

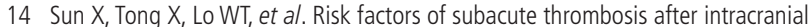
stenting for symptomatic intracranial arterial stenosis. Stroke 2017;48:784-6.

15 Shim EJ, Ryu CW, Park S, et al. Relationship between adverse events and antiplatelet drug resistance in neurovascular intervention: a meta-analysis. J Neurointerv Surg 2018:neurintsurg-2017-013632.

16 Oran I, Cinar C, Bozkaya H, et al. Tailoring platelet inhibition according to multiple electrode aggregometry decreases the rate of thrombotic complications after intracranial flow-diverting stent implantation. J Neurointerv Surg 2015;7:357-62.

17 Lee KH, Ahn Y, Kim SS, et al. Comparison of triple anti-platelet therapy and dual anti-platelet therapy in patients with acute myocardial infarction who had no-reflow phenomenon during percutaneous coronary intervention. Circ J 2013;77:2973-81.

18 Price MJ, Berger PB, Teirstein PS, et al. Standard-vs high-dose clopidogrel based on platelet function testing after percutaneous coronary intervention: the GRAVITAS randomized trial. JAMA 2011;305:1097-105.

19 Mehta SR, Tanguay JF, Eikelboom JW, et al. Double-dose versus standard-dose clopidogrel and high-dose versus low-dose aspirin in individuals undergoing percutaneous coronary intervention for acute coronary syndromes (CURRENT-OASIS 7): a randomised factorial trial. Lancet 2010;376:1233-43

20 Campbell CL, Smyth S, Montalescot G, et al. Aspirin dose for the prevention of cardiovascular disease: a systematic review. JAMA 2007;297:2018-24.

21 Andell $P$, James SK, Cannon CP, et al. Ticagrelor versus clopidogrel in patients with acute coronary syndromes and chronic obstructive pulmonary disease: an analysis from the platelet inhibition and patient outcomes (PLATO) trial. J Am Heart Assoc 2015:4:e002490.

22 Akbari SH, Reynolds MR, Kadkhodayan Y, et al. Hemorrhagic complications after prasugrel (Effient) therapy for vascular neurointerventional procedures. J Neurointerv Surg 2013;5:337-43.

23 Yang TH, Kim DI, Kim JY, et al. Comparison of triple anti-platelet therapy (aspirin, clopidogrel, and cilostazol) and double anti-platelet therapy (aspirin and clopidogrel) on platelet aggregation in type 2 diabetic patients undergoing drug-eluting stent implantation. Korean Circ J 2009;39:462-6.

24 Niazi AK, Dinicolantonio JJ, Lavie CJ, et al. Triple versus dual antiplatelet therapy in acute coronary syndromes: adding cilostazol to aspirin and clopidogrel? Cardiology 2013;126:233-43.

25 Fan ZG, Ding GB, Li XB, et al. The clinical outcomes of triple antiplatelet therapy versus dual antiplatelet therapy for high-risk patients after coronary stent implantation: a meta-analysis of 11 clinical trials and 9,553 patients. Drug Des Devel Ther 2016;10:3435-48.

26 Gao W, Zhang Q, Ge H, et al. Efficacy and safety of triple antiplatelet therapy in obese patients undergoing stent implantation. Angiology 2013;64:554-8.

27 Nakagawa I, Park HS, Wada T, et al. Efficacy of cilostazol-based dual antiplatelet treatment in patients undergoing carotid artery stenting. Neurol Res 2017:39:695-701.

28 Uchiyama S, Shinohara Y, Katayama Y, et al. Benefit of cilostazol in patients with high risk of bleeding: subanalysis of cilostazol stroke prevention study 2. Cerebrovasc Dis 2014:37:296-303

29 Takagi T, Hara H. Protective effects of cilostazol against hemorrhagic stroke: current and future perspectives. J Pharmaco/ Sci 2016;131:155-61.

30 Fiorella D, Derdeyn CP, Lynn MJ, et al. Detailed analysis of periprocedural strokes in patients undergoing intracranial stenting in Stenting and Aggressive Medical Management for Preventing Recurrent Stroke in Intracranial Stenosis (SAMMPRIS). Stroke 2012:43:2682-8

31 Tang YD, Wang W, Yang M, et al. Randomized comparisons of double-dose clopidogrel or adjunctive cilostazol versus standard dual anti-platelet in patients with high post-treatment platelet reactivity: Results of the creative trial (clopidogrel response evaluation and anti-platelet intervention in high thrombotic risk pci patients) Circulation 2018;137:2231-45. 